\title{
Erhöhte Leberwerte - Zufallsbefund in der Hausarztpraxis
}

\author{
Claudia Keerl und Christine Bernsmeier \\ Gastroenterologie und Hepatologie, Clarunis Universitäres Bauchzentrum Basel, St. Claraspital und Universitätsspital Basel \\ Claudia Keerl und Christine Bernsmeier haben in gleichem Masse zum Manuskript beigetragen
}

\begin{abstract}
Zusammenfassung: In der hausärztlichen Praxis finden sich im Rahmen der Abklärung unspezifischer Beschwerden oder während einer Gesundheitsvorsorgeuntersuchung bei 25\% aller untersuchten Patienten passager oder chronisch erhöhte Leberwerte. In der gesunden Bevölkerung zeigen sich erhöhte Leberwerte bei rund $2.5 \%$. Umgekehrt garantieren normale Leberwerte aber nicht immer eine gesunde Leber. Lebererkrankungen sind aber nicht nur häufig, sondern implizieren auch eine erhebliche Mortalität. Ihre Prävalenz nimmt stetig zu, und Lebererkrankungen stellen inzwischen die weltweit fünfthäufigste Todesursache dar. Führend in Prävalenz und verantwortlich für deren Anstieg sind insbesondere nicht-alkoholische Fettleber Erkrankung (NAFLD), alkohol-bedingte Leberschädigung (ARLD) und virale Hepatitiden. Wegen der Vielfalt möglicher Differenzialdiagnosen und dem gleichzeitigen Vorkommen mehrerer Ätiologien stellen sie eine diagnostische Herausforderung mit bedeutender prognostischer Implikation dar. Bei erhöhten Leberwerten sollten initial eine ausführliche Anamnese, sowie eine gründliche klinische Untersuchung durchgeführt werden. Hierdurch lassen sich mögliche Ursachen bereits auf wenige Differenzialdiagnosen eingrenzen. Anschliessend erfolgt je nach Ausmass der Leberwerterhöhung und klinischer Präsentation als Hepatitis oder cholestatischem Krankheitsbild ein Hepatopathiescreening. Die Labordiagnostik und obligate Ultraschalldiagnostik kann durch nicht-invasive Methoden (beispielweise Elastographie, Magnetresonanz-Cholangiopankreatikographie [MRI / MRCP], Computertomographie [CT]) und in ausgewählten Fällen durch invasive Methoden (Leberbiopsie, endoskopische retrograde Cholangiopankreatikographie [ERCP]) ergänzt werden. Im folgenden Artikel stellen wir die Abklärung erhöhter Leberwerte anhand von Algorithmen dar.
\end{abstract}

\section{Elevated liver function tests - as incidental finding in general practice}

\begin{abstract}
In general practice, elevated liver function tests are found in $25 \%$ of patients presenting with unspecific symptoms or during a routine health checkup. Approximately $2.5 \%$ of the general population are expected to show elevated values. Conversely, liver disease can present without abnormal liver function tests. Liver disease is not only frequent but may also imply a significant mortality. Worldwide its prevalence is steadily rising, and liver disease has become the fifth leading cause of death. Responsible for the rapidly increasing prevalence are primarily non-alcoholic fatty liver disease (NAFLD), alcohol-related liver disease (ARLD) and viral hepatitis. Due to the large range of possible differential diagnoses and the coexistence of aetiologies liver diseases represent a diagnostic challenge with important prognostic implication. In case of elevated liver function, a detailed medical history and a thorough clinical examination should be performed first. This allows narrowing down possible causes to the few most likely differential diagnoses. Subsequently, depending on the level of elevated liver function and the clinical presentation as hepatitis or cholestasis, screening tests for differential liver diseases are performed. Laboratory diagnostics and obligatory ultrasound scan can be supplemented by non-invasive methods (e.g. elastography, MRI / MRCP/CT) and in selected cases invasive methods (liver biopsy, ERCP). In the following article we delineate the diagnostic approach to elevated liver function tests using algorithms.
\end{abstract}

\section{Einführung}

Eine Abklärung erhöhter Leberwerte ist essenziell, um schwere, potenziell lebensbedrohliche Lebererkrankungen wie akutes Leberversagen, eine bereits bestehende chronische Lebererkrankung oder deren Spätfolgen bis hin zur Leberzirrhose zu erkennen oder möglicherweise $\mathrm{zu}$ verhindern. In der Praxis sind diese Abgrenzungen nicht immer leicht. Die Anzahl möglicher Differenzialdiagnosen und spezifischer Untersuchungen zu deren Nachweis bei Patienten mit erhöhten Leberwerten ist au- sserordentlich gross. Es ist auch zumeist nicht notwendig, bei allen Patienten mit erhöhten Leberwerten alle möglichen Differenzialdiagnosen auszuschliessen. Für den Arzt in der Praxis stellt sich die Frage nach der richtigen Auswahl und Sequenz an Blutuntersuchungen und ergänzender nicht-invasiver und invasiver Tests im klinischen Kontext. Durch Routinelabortests zusammen mit einer gezielten Anamnese und klinischen Untersuchung lässt sich die Vielzahl möglicher Differenzialdiagnosen und weiterer Untersuchungen bereits erheblich einschränken. 
Tabelle 1. Systematische Anamneseerhebung bei erhöhten Leberwerten zur Eingrenzung individueller Differentialdiagnosen

\begin{tabular}{|c|c|c|c|c|}
\hline Allgemein & Symptome & Komorbiditäten & Medikamente & Familienanamnese \\
\hline $\begin{array}{l}\text { Alter } \\
\text { Ethnie }\end{array}$ & $\begin{array}{l}\text { Ikerus } \\
\text { Juckreiz } \\
\text { Lehmstuhl } \\
\text { Dunkler Urin }\end{array}$ & $\begin{array}{l}\text { Autoimmunerkrankungen } \\
\text { (z.B. Thyreopathien, } \\
\text { chronisch entzündliche } \\
\text { Darmerkrankungen, } \\
\text { Diabetes mellitus, Zöliakie } \\
\text { etc.) }\end{array}$ & $\begin{array}{l}\text { Aktuelle Medikation } \\
\text { (insbesondere: Antibiotika, } \\
\text { Analgetika, Antikonvulsiva, } \\
\text { Antirheumatika, } \\
\text { Antihypertensia etc.) }\end{array}$ & $\begin{array}{l}\text { Hereditäre Lebererkrank- } \\
\text { ungen (Hämochromatose / } \\
\text { M. Wilson/alpha-1 } \\
\text { Antitrypsinmangel) }\end{array}$ \\
\hline $\begin{array}{l}\text { Operationen, } \\
\text { Tätowierungen } \\
\text { (Ort der Durchführung) }\end{array}$ & $\begin{array}{l}\text { (Abdominal) Schmerz } \\
\text { Nausea }\end{array}$ & Metabolisches Syndrom & Phytotherapeutika & Virale Hepatitiden \\
\hline $\begin{array}{l}\text { Bluttransfusionen } \\
\text { (insbesondere vor 1992) }\end{array}$ & $\begin{array}{l}\text { Spider nävi } \\
\text { Palmarerythem }\end{array}$ & $\begin{array}{l}\text { kardiale, pulmonale } \\
\text { Erkrankungen }\end{array}$ & $\begin{array}{l}\text { «Over-the-counter» } \\
\text { Präparate }\end{array}$ & $\begin{array}{l}\text { Hepatocelluäres/ } \\
\text { Cholangiozelluläres } \\
\text { Karzinom }\end{array}$ \\
\hline $\begin{array}{l}\text { Risikoverhalten (Drogen, } \\
\text { Alkohol, Sexualverhalten) }\end{array}$ & $\begin{array}{l}\text { Fatigue } \\
\text { Verwirrtheitszustände }\end{array}$ & $\begin{array}{l}\text { Hämatologische } \\
\text { Erkrankungen }\end{array}$ & Chinesische Medizin/ Tees & $\begin{array}{l}\text { Diabetes mellitus/ } \\
\text { Metabolisches Syndrom }\end{array}$ \\
\hline $\begin{array}{l}\text { Verzehr (selbstgesammel- } \\
\text { ter) Pilze, Wildgerichte, } \\
\text { ungenügend gegartes } \\
\text { Fleisch oder Fisch, } \\
\text { Schalentiere }\end{array}$ & $\begin{array}{l}\text { Gewichtsverlust } \\
\text { Fieber }\end{array}$ & & $\begin{array}{l}\text { «Life Style Präparate» } \\
\text { (Ernährungsergänzungen, } \\
\text { Diätpräparate, Anabolika } \\
\text { etc.) }\end{array}$ & \\
\hline Reiseanamnese & $\begin{array}{l}\text { Meläna } \\
\text { Hämatemesis }\end{array}$ & & $\begin{array}{l}\text { Dauer / Dosis / Start } \\
\text { und Stopp }\end{array}$ & \\
\hline
\end{tabular}

Auf den folgenden Seiten wird versucht aufgrund der unterschiedlichen personellen und infrastrukturellen Voraussetzungen von Hausarztpraxen, Notfallstationen und gastroenterologisch/hepatologischer Abteilungen ein situationsbezogener Leitfaden zur Abklärung erhöhter Leberwerte zu erstellen.

\section{Diagnostik}

\section{Anamnese und klinische Untersuchung}

Die Erhebung einer umfassenden und gezielten Anamnese ist evident und führt zumeist bereits zu einer Verdachtsdiagnose beziehungsweise engt das differenzialdiagnostische Spektrum ein.

$\mathrm{Zu}$ einer vollständigen Anamnese gehören unter anderem eine ausführliche persönliche Anamnese inklusive bisheriger Krankengeschichte, Medikamente (verschreibungspflichtige Medikamente, «Over-the-Counter» drugs, Phytotherapeutika, Homöopathie, chinesische Heilmittel und Tees, Nahrungsergänzungsmittel), Noxen (Alkohol, Nikotin, Drogen), Toxine, diverse Risikofaktoren und eine Familienanamnese. Eine detaillierte Zusammenstellung findet sich in Tabelle 1.

Die körperliche Untersuchung sollte fokussiert sein auf Zeichen, die mit Lebererkrankungen assoziiert sind: BodyMass-Index (BMI), Hepatosplenomegalie, Aszites, Ödeme, Sarkopenie, Gynäkomastie, Leberhautzeichen (Palmarerythem, Spider nävi), gestaute Halsvenen oder psychomotorische Verlangsamung.

\section{Labor}

\section{Bei welchen Patienten sollten die Leberwerte bestimmt werden?}

Die meisten Lebererkrankungen entwickeln sich langsam und asymptomatisch oder mit unspezifischen Symptomen wie Fatigue, Übelkeit, Juckreiz oder Gewichtsverlust [1], so dass diese Symptome ein möglicher Grund für eine Leberwertkontrolle sein können. Typische Symptome einer Leberzirrhose treten meist erst bei fortgeschrittener Erkrankung mit klinisch signifikanter portaler Hypertonie oder bei akut-auf-chronischem Leberversagen auf und sollten dann umgehend durch einen Facharzt für Gastroenterologie oder Hepatologie evaluiert werden.

Bei Patienten mit Insulinresistenz oder metabolischen Risikofaktoren (Übergewicht, Adipositas, Diabetes, arterielle Hypertonie, Dyslipidämie, koronare Herzkrankheit [KHK]) sollte nach einer nicht-alkoholische Fettleber Erkrankung (NAFLD) durch Bestimmung der Leberwerte und Durchführung eines Ultraschalls mit Frage nach Steatose gesucht werden.

Bei Patienten, die potenziell hepatotoxische Medikamente einnehmen, bei Patienten mit Autoimmunkrankheiten und bei positiver Familienanamnese für hepatologische Erkrankungen ist eine Bestimmung der Leberwerte sinnvoll. Bei Prädisposition zu genetischen Erkrankungen (z.B. Hämochromatose, M. Wilson) sind gegebenenfalls auch spezifische Tests indiziert.

Patienten mit einem Hochrisikoprofil für virale Hepatitiden (Drogenabusus, Patienten aus Hochrisikogebieten, HIV [Humanes Immundefizienz-Virus] infizierte, MSM [men who have sex with men], Gefängnisinsassen und andere) sollten hierauf gescreent werden. Vor dem Hinter- 
Tabelle 2. Standard- und erweitertes Hepatopathiescreening bei asymptomatischer Leberwerterhöhung

\begin{tabular}{|c|c|c|}
\hline & Standard Screening & Erweitertes Screening \\
\hline viral & $\begin{array}{l}\text { HBs Antigen (HBsAg), HBc Antikörper (Ak) } \\
\text { HCV Ak } \\
\text { Hepatitis E (HEV) Ak, HEV PCR (bei V.a. akute } \\
\text { Infektion/ Immunsuppression) }\end{array}$ & $\begin{array}{l}\text { HBs Ak, HBV DNA, HDV Ak } \\
\text { HCV PCR }\end{array}$ \\
\hline Eisenstatus & Ferritin und Transferrinsättigung & $\begin{array}{l}\text { HFE-Gen Mutation } \\
\text { (hereditäres-Hämochromatose Protein) }\end{array}$ \\
\hline autoimmun & $\begin{array}{l}\text { anti-nucleare Antikörper (ANA), anti-smooth } \\
\text { muscle Antikörper (SMA) } \\
\text { anti-mitochondriale Antikörper (AMA-M2) } \\
\text { Serumimmunglobuline (IgG, IgM) }\end{array}$ & $\begin{array}{l}\text { anti-Leber-Niere-Mikrosomen Antikörper (LKM1) } \\
\text { anti-neutrophile cytoplasmatische Antikörper (ANCA) } \\
\text { Sprueserologie (anti-tissue Transglutaminase IgA, Gesamt-IgA) }\end{array}$ \\
\hline metabolisch & thyreotropes Hormon (TSH) & $\begin{array}{l}\text { Alpha-1 Antitrypsin } \\
\text { Coeruloplasmin }\end{array}$ \\
\hline Tumormarker & & $\begin{array}{l}\text { Alpha-1-Fetoprotein (AFP) } \\
\text { Carcinoembryonales Antigen (CEA) } \\
\text { Carbohydrate Antigen (CA 19-9) }\end{array}$ \\
\hline
\end{tabular}

grund epidemiologischer Veränderungen mit deutlich steigender Prävalenz der Hepatitis C (HCV) Infektionen wurde zuletzt durch die AASLD (American Association for the Study of Liver Diseases) ein einmaliges HCV-Screening für alle Personen ab dem 18. Lebensjahr, sowie Personen unter 18 Jahren mit Risikoverhalten empfohlen [2].

\section{Was sind die aktuellen Normwerte und was gilt als erhöht?}

Häufig wird angenommen, dass leicht erhöhte Leberwerte einer vorübergehenden oder zufälligen Fluktuation entsprechen, sich innerhalb kurzer Frist normalisieren und somit keine schwerwiegende Lebererkrankung darstellen. Dies mag für Begleithepatitiden und milde Verläufe akuter Hepatitiden (z.B. medikamentös-toxische Hepatitiden) im Einzelfall stimmen - jedoch nicht für chronische Lebererkrankungen. Bei den am häufigsten vorkommenden chronischen Lebererkrankungen in der Schweiz (NAFLD, ARLD, Virushepatitiden [Hepatitis B Virus, HBV, und HCV]), sind die Leberwerte häufig normal oder nur leicht erhöht.

Die Referenzen für Leberwerte, die wir heute kennen, wurden bereits in den 1950er Jahren entwickelt und festgelegt. Vermutlich sind die aktuellen oberen Normwerte zu hoch, da damals auch Patienten mit NAFLD in die Gruppe zur Bestimmung des Referenzbereiches eingeschlossen wurden $[3,4]$. Sowohl Leberwerte im Normbereich als auch oberhalb der Norm entsprechen nicht notwendigerweise ihrer klinischen Signifikanz. Diese Diskrepanz kann dazu führen, dass einerseits bei erhöhten Werten ohne klinische Signifikanz inadäquate und teure Abklärungen in die Wege geleitet werden, und andererseits bei normalen Leberwerten bestehende chronische Hepatopathien unterdiagnostiziert bleiben. Die ALFIE Studie hatte gezeigt, dass lediglich bei $50 \%$ der auffälligen Leberwerttests überhaupt ein Follow-Up erfolgte und bei $50 \%$ der neu diagnostizierten fortgeschrittenen Lebererkrankungen bereits im Vorfeld der Diagnosestellung erhöhte Leberwerte gemessen worden waren, ohne dass weitere Untersuchungen in die Wege geleitet wurden [5].
Umso wichtiger ist daher die Einordnung der Leberwerte in die Gesamtsituation des Patienten. Diese sollten immer in Rückschau auf die Krankengeschichte des Patienten, die vergangene und aktuelle Medikation und in Bezug auf vorhandene Vorwerte interpretiert werden.

\section{Sollten erhöhte Leberwerte zunächst kontrolliert werden?}

Die BALLETS Studie hatte gezeigt, dass die Leberwerte bei Wiederholung nach vier Wochen bei $84 \%$ der Patienten erhöht blieben [6]. Aus gesundheits-ökonomischer Sicht ist eine Wiederholung nur bei hoher Wahrscheinlichkeit für eine Normalisierung sinnvoll. Die Guidelines der British Society of Gastroenterology (BSG) empfehlen im Gegensatz zu den amerikanischen Guidelines stets die Durchführung eines Basis Hepatopathie Screenings bei erhöhten Leberwerten unabhängig von Ausmass und Dauer [7]. Es kann also bei leicht erhöhten Leberwerten je nach klinischem Kontext (z.B. nach kürzlich verabreichte passagere Antibiotikatherapie, akuter Infektion) vertretbar sein, eine kurzfristige Kontrolle durchzuführen, sollten die Werte jedoch erhöht bleiben oder sogar ansteigen, muss eine Abklärung erfolgen.

\section{Welche Werte sollen getestet werden?}

Die meisten Labore im ambulanten und stationären Bereich bieten fertige Laborblöcke zur Bestimmung von Leberwerten an. $\mathrm{Zu}$ unterscheiden sind Leberenzymbestimmungen (Aspartat-Aminotransferase [AST], AlaninAminotransferase [ALT], alkalische Phosphatase [AP] und Gamma-Glutamyl Transferase [GGT]) von Lebersyntheseleistungstests (Bilirubin, Albumin und Quick/INR). Initial scheint es sinnvoll folgende Werte in der Routine zu bestimmen: ALT, AP und GGT sowie Albumin, Bilirubin und INR zusätzlich zu einem Differenzialblutbild und C-reaktivem Protein (CRP). Bei einer nicht-akuten asymptomatischen Leberwerterhöhung sollte entsprechend Empfehlungen der BSG zusätzlich zu einem transabdominellen Ultraschall ein Standard-Hepatopathiescreening erfolgen, welches je nach Befund in einem zweiten Schritt erweitert werden kann 
(siehe Tab. 2) [7]. Bei einer akuten symptomatischen Hepatopathie hingegen sollten alle differenzialdiagnostischen Ätiologien bereits zu Anfang gesucht werden.

\section{Transaminasen (AST und ALT)}

Die Enzyme AST und ALT sind nicht leberspezifisch, da sie neben der Leber auch in anderen Geweben vorhanden sind. Die ALT ist etwas leberspezifischer, da sie extrahepatisch nur in geringer Konzentration vorkommt. Das Enzym AST, weniger auch die ALT, finden sich unter anderem auch im skelettalen und kardialen Muskelgewebe, und können somit auch bei Muskel- oder Herzerkrankungen erhöht sein. Bei schwerer Leberschädigung, z. B. im Rahmen einer alkoholinduzierten oder toxischen Hepatitis, ist häufig die AST höher als die ALT. Sind AST und / oder ALT im Rahmen einer Leberschädigung im Vergleich zur alkalischen Phosphatase (AP) deutlich erhöht, spricht man von einem hepatozellulären Muster. Differenzialdiagnostische Überlegungen in Zusammenhang mit der Höhe der Transaminasen finden Sie in Tabelle 3.

Für das Vorgehen in der Praxis empfiehlt sich die Anwendung von Algorithmen in Abhängigkeit des Leberwertmusters und der vorherrschenden Klinik (Abb. 1 und 2).

\section{Alkalische Phosphatase (AP)}

Die AP wird beim Erwachsenen zum Grossteil in der Leber, jedoch in geringeren Mengen auch in Knochen, Darm, Nieren und Leukozyten gebildet. Zur Bestätigung des hepatischen Ursprungs der AP sollten zusätzlich immer GGT und Bilirubin bestimmt werden [8]. Bei einer disproportional erhöhten AP im Vergleich zu AST / ALT spricht man von einem cholestatischen Schädigungsmuster. Ist die GGT nicht begleitend erhöht, so muss von einem extrahepatischen Ursprung der Enzymerhöhung ausgegangen wer-

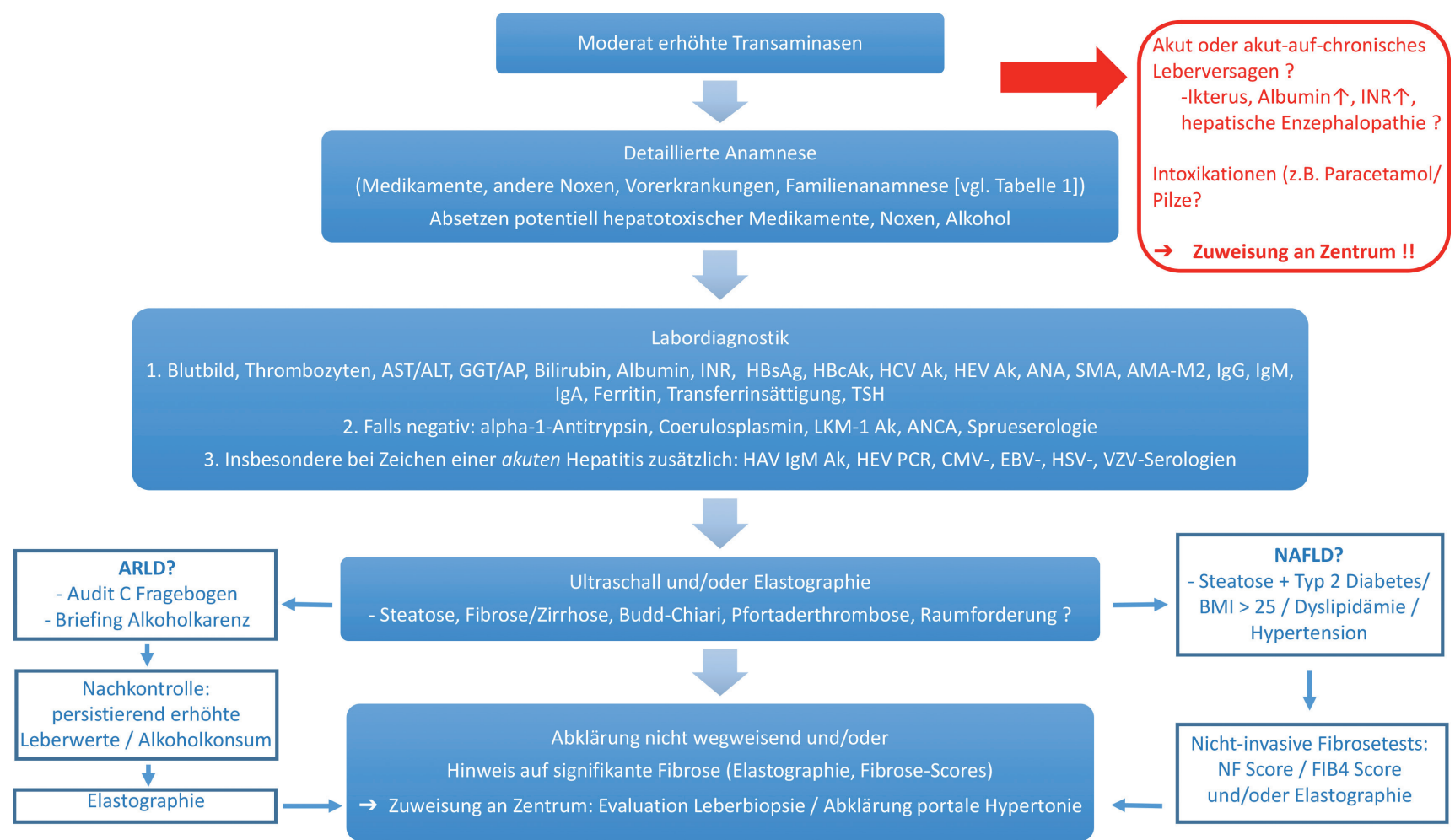

Abbildung 1. Algorithmus zur Abklärung moderat erhöhter Transaminasen.

Tabelle 3. Differentialdiagnose erhöhter Transaminasen

\begin{tabular}{|c|c|c|c|}
\hline leicht erhöhte Transaminsean & $\begin{array}{l}\text { moderat erhöhte Transaminasen } \\
(>100 \mathrm{U} / \mathrm{l})\end{array}$ & Transaminasen $>1000 \mathrm{U} / \mathrm{l}$ & $\begin{array}{l}\text { erhöhte Transaminasen - } \\
\text { extrahepatische Ursachen }\end{array}$ \\
\hline Zirrhose & Autoimmunhepatitis & Ischämische Hepatitis & $\begin{array}{l}\text { Muskelerkrankungen } \\
\text { inkl. Rhabdomyolyse }\end{array}$ \\
\hline $\begin{array}{l}\text { Alkoholische Hepatopathie } \\
\text { (ARLD) }\end{array}$ & Alkoholische Hepatitis (Flare) & $\begin{array}{l}\text { Toxische Hepatitis (Vergiftungen, } \\
\text { Drogen, Medikamente) }\end{array}$ & $\begin{array}{l}\text { Herzmuskelschaden } \\
\text { (Herzinfarkt und andere) }\end{array}$ \\
\hline $\begin{array}{l}\text { Nicht-alkoholische Fettleber } \\
\text { Erkrankung (NAFLD) }\end{array}$ & $\begin{array}{l}\text { Akute Virushepatitiden (HAV, HBV, } \\
\text { HCV, HDV, HEV, CMV, EBV, VZV, HSV) }\end{array}$ & $\begin{array}{l}\text { Akute Virushepatitiden (HAV, HBV, } \\
\text { HCV, HDV, HEV, CMV, EBV, VZV, HSV) }\end{array}$ & $\begin{array}{l}\text { Hyper-/Hypothyreose } \\
\text { Nebenniereninsuffizienz }\end{array}$ \\
\hline $\begin{array}{l}\text { Chronische Virushepatitis } \\
(\mathrm{HCV}, \mathrm{HBV}, \mathrm{HDV}, \mathrm{HEV})\end{array}$ & $\begin{array}{l}\text { Toxische Hepatopathien } \\
\text { (Vergiftungen, Medikamente) }\end{array}$ & & «Makro-AST» \\
\hline $\begin{array}{l}\text { andere chronische } \\
\text { Hepatopathien }\end{array}$ & & & Hitzeschaden \\
\hline
\end{tabular}


den. Die häufigsten Ursachen sind Vitamin-D-Mangel, die Wachstumsphase bei Kindern und Jugendlichen, erhöhter Knochenstoffwechsel in den Wechseljahren sowie in der Schwangerschaft; seltener Knochenmetastasen oder Morbus Paget [7]. Bei Patienten mit der Blutgruppe O und B kann die AP nach einer fettreichen Mahlzeit [9] erhöht sein aufgrund erhöhter intestinaler AP.

\section{Gamma-Glutamyltransferase (GGT)}

Die GGT ist unspezifisch und findet sich ebenfalls in zahlreichen Geweben (Leber, Niere, Prostata, Darm, Pankreas). Bei einer isolierten Erhöhung ohne begleitende erhöhte alkalische Phosphatase liegt zumeist eine Enzyminduktion durch Medikamente, Alkoholkonsum oder im Rahmen eines metabolischen Syndroms vor. Die GGT kann jedoch auch bei Pankreaserkrankungen, Niereninsuffizienz, Lungenemphysem oder anderen Erkrankungen erhöht sein. Die Bestimmung der GGT dient nicht nur der Bestätigung des hepatischen Ursprungs einer erhöhten AP, sondern ist auch im Rahmen vieler Hepatitiden begleitend erhöht.

\section{Bilirubin}

Bei der Messung von Bilirubin können «unkonjugiertes» (indirektes) und «konjugiertes» (direktes) Bilirubin unterschieden werden. Gleichzeitig mit anderen Leberwerten (insbesondere einer erhöhten AP) findet sich ein erhöhtes Bilirubin einerseits bei sonographisch erweiterten Gallenwegen bei einer extrahepatischen Cholestase z.B. durch Konkremente, benigne oder maligne Gallengangsste- nosen. Ohne Gallenganserweiterung liegt bei einer Erhöhung des Bilirubins eine intrahepatische Cholestase (z.B. bei einer fortgeschrittenen Leberzirrhose, schweren Hepatitis oder cholestatischen Hepatopathie) vor. Seltene Ursache einer isolierten direkten Hyperbilirubinämie ohne bildgebenden Hinweis auf extrahepatische Ursache sind angeborene Enzymdefekte mit meist guter Prognose (Dubin-Johnson, Rotor Syndrom). Ein erhöhtes indirektes Bilirubin ist zumeist bedingt durch eine Überproduktion (z.B. eine Hämolyse), eine verminderte intrahepatische Aufnahme oder eine verminderte intrahepatische Konjugation. Die häufigste Form einer isolierten indirekten Hyperbilirubinämie ist das Gilbert Syndrom (Morbus Meulengracht). Es betrifft etwa 3-7\% der Bevölkerung [3]. Bei asymptomatischen, gesunden Patienten mit milder indirekter Hyperbilirubinämie und normwertigen Transaminasen, negativer Medikamentenanamnese ohne Hinweise auf eine Hämolyse darf die Diagnose eines Morbus Meulengracht ohne genetische Diagnostik gestellt werden. Auch Fasten kann das indirekte Bilirubin um das Zwei- bis Dreifache erhöhen.

Zur Abklärung eines cholestastischen Leberenzymmusters kann folgender Algorithmus (Abb. 3) angewandt werden. Bei bestätigtem hepatischem Ursprung der AP kann je nach sonographischem Befund zwischen einer intraund extrahepatischer Cholestase bzw. einer Leberläsion unterschieden werden. In Tabelle 4 werden die Differenzialdiagnosen bei erhöhter AP hepatischen und nicht hepatischen Ursprungs aufgelistet.

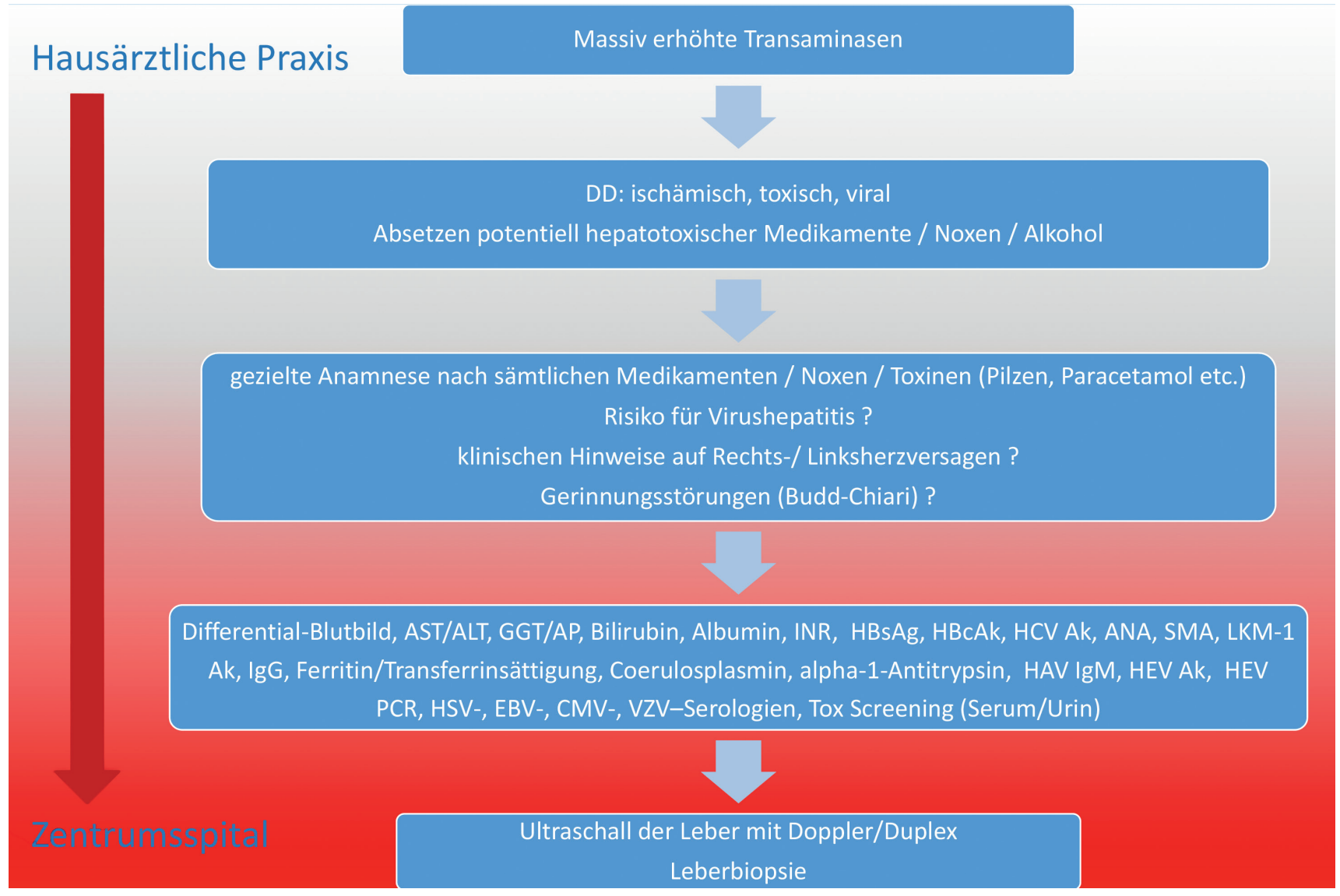

Abbildung 2. Algorithmus zur Abklärung massiv erhöhter Transaminasen (ALT > 1000 IU / I). 
Tabelle 4. Differentialdiagnose erhöhter cholestatischer Leberwerte

\begin{tabular}{|c|c|c|}
\hline Hepatozelluläre Cholestase & Cholangiozelluläre Cholestase & Obstruktive Cholestase \\
\hline Leberzirrhose & Primäre biliäre Cholangitis (PBC) & Choledocholithiasis, Hepatolithiasis \\
\hline Alkoholische und nicht-alkoholische Steatohepatitis & $\begin{array}{l}\text { Primär (und sekundär) sklerosierende } \\
\text { Cholangitis (PSC) }\end{array}$ & Cholangiokarzinom (extrahepatisch) \\
\hline Drug induced liver injury (DILI, cholestatische Form) & IgG4-assozierte Cholangitis & $\begin{array}{l}\text { Pankreaskopf-, Gallenblasen-, } \\
\text { Papillenkarzinom }\end{array}$ \\
\hline $\begin{array}{l}\text { Maligne infiltrative Erkrankungen } \\
\text { (z.B. Hepatozelluläres Karzinom, Lymphome, andere) }\end{array}$ & Drug induced Cholangiopathie (DILI) & PSC (signifikante Stenose) \\
\hline $\begin{array}{l}\text { Benigne infiltrative Erkrankungen (Sarkoidose, } \\
\text { Amyloidose, Tuberkulose) }\end{array}$ & Cholangiokarzinom (intrahepatisch) & Pankreatitis, Pankreaspseudozysten \\
\hline Sepsis & Cystische Fibrose & Metastasen, Lymphknotenmetastasen \\
\hline Virale Hepatitis & Graft vs. Host Disease & Duodenaldivertikel \\
\hline $\begin{array}{l}\text { (mono-) genetische Erkrankungen (BRIC 1-3, } \\
\text { PFIC 1-3, ICP, erythropoietische Protoporphyrie) }\end{array}$ & $\begin{array}{l}\text { Duktalplatten-Malformationen } \\
\text { (v. Meyenburg Komplexe, Caroli Syndrom, } \\
\text { kongenitale Leberfibrose) }\end{array}$ & Parasitosen (Fasziolosis, Askaridiasis) \\
\hline Nodulär regenerative Hyperplasie (NRH) & Idiopathische Duktopenie & Mirizzi Syndrom \\
\hline $\begin{array}{l}\text { Vaskuläre Erkrankungen (Stauungshepatopathie, } \\
\text { Budd-Chiari, sinusoidales Obstruktionssyndrom) }\end{array}$ & Langerhanszell Histiozytose & $\begin{array}{l}\text { Infektionen (AIDS Cholangiopathie, CMV, } \\
\text { Cryptosporidiosis, Mikrosporidiosis) }\end{array}$ \\
\hline Total parenterale Ernährung & & Gallengangsatresie \\
\hline Paraneoplastisch M. Hodgkin, Nierenzellkarzinom) & & \\
\hline
\end{tabular}

Anmerkungen: BRIC: benign recurrent intrahepatic cholestasis type 1-3; PFIC: progressive familial intrahepatic cholestasis type 1-3; ICP: Intrahepatische Schwangerschaftscholestase.

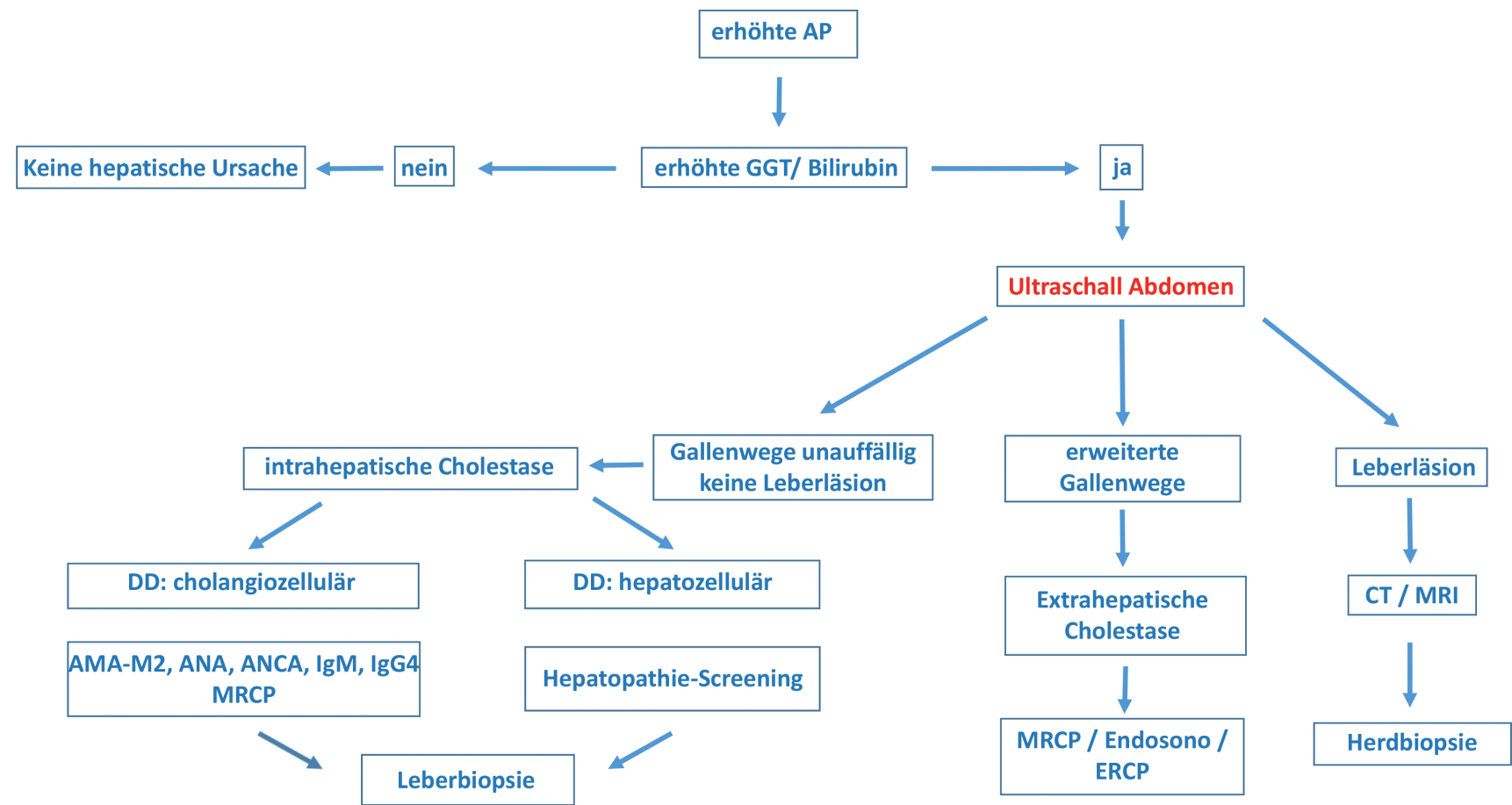

Abbildung 3. Algorithmus zur Abklärung cholestatischer Enzymmuster.

\section{Ferritin}

Ein erhöhtes Ferritin muss nicht immer Hinweis auf eine zugrundeliegende Hämochromatose sein, sondern findet sich auch bei diversen entzündlichen, metabolischen oder neoplastischen Krankheitsprozessen, in Zusammenhang mit erhöhten Leberwerten häufig bei ARLD und NAFLD.
Lebersyntheseleistungstests

Zur Einschätzung der Lebersyntheseleistung wird in erster Linie Albumin bestimmt, da dieses Protein nur in der Leber gebildet wird. Es hat eine Halbwertszeit von drei Wochen [8]. Albuminwerte unter $35 \mathrm{~g} / 1$ weisen auf eine Leberschädigung von mehr als drei Wochen hin. Jedoch sind tiefe Al- 
buminwerte unspezifisch und können auch bei verminderter Proteinaufnahme (Malabsorption), verminderter Synthese oder Verbrauch von Eiweiss (z. B. im Rahmen von SIRS [Systemic Inflammatory Response Syndrom] oder Sepsis) oder Verlust von Eiweiss (z.B. nephrotisches Syndrom) auftreten.

Sensitiver kann die Abschätzung der gegenwärtigen Syntheseleistung durch die Bestimmung von Gerinnungstests (Prothrombinzeit und INR) erfolgen, da die Faktoren II, V, VII, IX und X in der Leber gebildet werden. Eine Verlängerung der Prothrombinzeit zeigt sich bereits innerhalb von 24 Stunden nach schwerer Leberschädigung [8]. Die Faktoren II, VII, IX und X werden Vitamin-K-abhängig gebildet, und können bei einer Fettmalabsorption oder cholestatischen Lebererkrankungen vermindert sein. Eine Verlängerung der Prothrombinzeit kann natürlich auch viele andere Ursachen haben (z.B. PhenoprocoumonEinnahme, Heparin-Therapie, disseminierte intravasale Koagulopathie, Hypothermie) [3]. Spezifischer ist der Faktor $\mathrm{V}$ welcher in der Leber Vitamin-K-unabhängig gebildet wird.

\section{Bildgebung}

Als Bildgebung stehen je nach Fragestellung verschiedene Möglichkeiten zur Verfügung. Die Abdomensonographie ist immer die erste Modalität, da sie ubiquitär verfügbar, ungefährlich und kosteneffizient ist. Durch den Ultraschall lassen sich bereits viele Diagnosen stellen bzw. ausschliessen (Zeichen der Leberzirrhose, fokale Läsionen, intra- und extrahepatisch erweitere Gallenwege, vaskuläre Erkrankungen der Leber wie beispielsweise Pfortaderthrombose oder Budd-Chiari Syndrom, Aszites, Splenomegalie). Ergänzend ist die Möglichkeit der Quantifizierung der Fibrose mittels transienter Elastographie möglich [10] (siehe Nichtinvasive Leberfibrosetests). Bei speziellen Fragestellungen (z.B. Herdbefunden der Leber/Gallenwege/Pankreas, Gallenwegsobstruktion, primär sklerosierende Cholangitis [PSC], primär biliären Cholangitis [PBC], Choledocholithiasis, Cholangiokarzinom) sollte ergänzend eine Schnittbildgebung erfolgen (MRI mit Primovist, MRCP oder CT).

\section{Nicht-invasive Leberfibrosetests}

Nicht-invasive Methoden zur quantitativen Beurteilung der Fibrose wurden gut bei viralen Hepatitiden (HBV, HCV), und auch NAFLD und ARLD evaluiert [10]. Es stehen diverse serologische Biomarker (NAFLD Fibrosis Score [NFS], Fibrosis-4 [FIB-4] und andere) als auch Messmethoden der Lebersteifigkeit (transiente Elastograhie z.B. FibroScan ${ }^{\odot}$ ) zur Verfügung. Die Sensitivität dieser Methoden ist hoch bei fehlender Fibrose sowie bei Zirrhose, jedoch gering bei intermediären Fibrosestadien. Beim FibroScan ${ }^{\odot}$ finden sich falsch hohe Werte unter anderem bei einer floriden Hepatitis oder extrahepatischen Cholestase. Bei Aszites, Adipositas oder schmalen Interkostal- räumen kann die Messung oft nicht erfolgen. Zu beachten sind zudem die je nach Ätiologie unterschiedlichen Cutoff-Werte [10]. Die serologischen Marker sollten nüchtern evaluiert werden, da falsch positive Ergebnisse postprandial, jedoch auch bei Hämolyse, chronischen Entzündungen, unter HAART (highly active antiretroviral therapy), und akuter Hepatitis beschrieben wurden [10]. Für NAFLD ist in erster Linie eine Evaluation mittels NFS [12] oder FIB-4 [11] indiziert. Dies sind einfache Parameter, die schnell und kosteneffizient in jeder hausärztlichen Praxis bestimmt werden, und Patienten ohne Risiko für Leberfibrose identifizieren können (hoher negativ prädiktiver Wert). Die Berechnung erfolgt mit Hilfe von Apps (z. B. $\mathrm{HepCalc}^{\odot}, \mathrm{MedCalc}^{\odot}$ oder andere), welche teilweise kostenlos auf dem Smartphone installiert werden können.

\section{Leberbiopsie}

Wenn die serologischen und bildgebenden Abklärungen nicht zu einer eindeutigen Diagnose führen, das Ausmass der bereits eingetretenen Leberschädigung definiert werden soll (Entzündung = Grading; Fibrose $=$ Staging) oder mehrere Ursachen gleichzeitig für die Leberschädigung in Frage kommen, ist die Durchführung einer Biopsie indiziert. Die Leberbiopsie kann diverse qualitative Informationen über die Hepatopathie und deren Ätiologie liefern sowie Entzündung und Fibrose quantifizieren und gilt daher als Goldstandard für die Diagnostik von Lebererkrankungen. Die Indikation zur Leberbiopsie sollte gestellt werden, wenn sich hieraus eine therapeutische Konsequenz ergibt. Das Risiko einer Blutungskomplikation nach transkutaner Leberbiopsie liegt bei $0.7 \%$, bei $0.5 \%$ bedarf es einer Intervention (Transfusion / Coiling des blutenden Gefässes) [13].

\section{Wer sollte zum Spezialisten?}

\section{Akute Hepatopathien}

Patienten mit einer akuten Hepatitis mit massiver Erhöhung der Leberenzyme (vgl. Abb. 2), einer spontan erhöhten Prothrombinzeit/INR und/oder Enzephalopathie sollten umgehend einem Zentrum mit hepatologischer Abteilung zugewiesen werden.

\section{Chronische Hepatopathien mit möglicher signifikanter} Fibroseentwicklung

Generell sollten Patienten mit Verdacht auf oder nachgewiesener Hepatitis B (HBsAg positiv) oder C (HCV AK und HCV PCR positiv), Autoimmunhepatitis (erhöhte IgG +/Auto-Antikörper), PBC (AMA-M2 AK positiv, cholestastisches Leberenzymmuster), PSC (MRCP Befund, cholestatisches Muser + /-chronisch entzündliche Darmerkrankung), Hämochromatose (erhöhtes Ferritin und erhöhte Transferrinsättigung $>45 \%$ ) zumindest einmalig einem Spezialisten konsiliarisch vorgestellt werden. Weiterhin Patienten mit NALFD und V.a. signifikante Fibrose in einem der beschriebenen nicht-invasiven Tests [14]. 


\section{Alkoholkranke Patienten}

Zuweisung von alkoholkranken Patienten (Abklärung mittels AUDIT-Score) mit und ohne ARLD sollten zu einem Suchtspezialisten und ggf. auch Gastroenterologen / Heptologen.

\section{Wenn alle Untersuchungen unauffällig bleiben}

Findet sich trotz sorgfältiger Abklärungen keine Ursache für die Leberwerterhöhung, sollte unbedingt die Medikamentenanamnese inkl. der Phytotherapeutika wiederholt werden. Wichtig ist auch zu bedenken, dass der Ultraschall erst für eine Steatose von mindestens $20 \%$ der Hepatozyten sensitiv ist [15], sodass Patienten mit unauffälligen Ultraschall und metabolischen Syndrom dennoch an einer NAFLD leiden können und somit in den Abklärungsalgorithmus mit Frage nach signifikanter Fibrose eintreten sollten.

Bei Patienten mit persistierend erhöhten Leberwerten ohne erklärbare Ursache sollte unbedingt eine Zuweisung zum Spezialisten erfolgen, um die erfolgten Abklärungen ggf. zu ergänzen, eine invasive Diagnostik zu evaluieren (Leberbiopsie und/oder ERCP) und damit relevante Lebererkrankungen nicht zu übersehen.

\section{Fazit}

Da nahezu jede Basisdiagnostik heutzutage Leberparameter enthält, ist man in der hausärztlichen Praxis im Alltag regelmässig mit erhöhten Werten konfrontiert. Oft besteht Unsicherheit bezüglich der Notwendigkeit, diese abzuklären, dem Zeitraum und Umfang. Hepatopathien sind häufig, gehen mit einer hohen Morbidität und Mortalität einher und sind in vielen Fällen behandelbar oder sogar heilbar, weshalb die Ätiologie unbedingt gesucht werden sollte. Nach gezielter Anamnese und körperlicher Untersuchung mit Fokus auf klinische Zeichen einer Hepatopathie kann häufig bereits eine Eingrenzung möglicher Differenzialdiagnosen erfolgen. Die häufigsten Gründe erhöhter Leberwerte sind eine NAFLD oder ARLD, hepatotoxische Medikamente und virale Hepatitiden. Seltenere Ursachen sind genetische Speichererkrankungen wie beispielsweise eine Hämochromatose oder autoimmune Hepatopathien (Autoimmunhepatitis, PBC, PSC). Nach Abnahme einer hepatologischen Basisdiagnostik erfolgt in Abhängigkeit der Höhe der Leberwerte sowie des Enzymmusters eine weiterführende Stufendiagnostik anhand verschiedener Algorithmen. Bei Hinweisen auf eine chronische Hepatopathie mit Zeichen der signifikanten Fibrose und unklaren Hepatopathien sollte die Zuweisung $\mathrm{zu}$ einem Facharzt für Gastroenterologie und Hepatologie erfolgen. Dieser kann ggf. weitere gezielte Abklärungsschritte beispielsweise eine Elastographie, Leberbiopsie, MRCP oder ERCP in die Wege leiten. Bestehen Hinweise auf eine akute oder akut-auf-chronische Hepatopathie mit Zeichen einer Leberinsuffizienz ist die dringliche Zuweisung an ein Zentrum geboten.

\section{Literatur}

1. Williams R, Ashton K, Aspinall R, Bellis MA, Bosanquet J, Cramp $\mathrm{ME}$, et al. Implementation of the Lancet Standing Commission on Liver Disease in the UK. Lancet. 2015; 386: 2098-111.

2. Ghany MG, Morgan TR. Hepatitis C Guidance 2019 Update: American Association for the Study of Liver Diseases - Infectious Diseases Society of America Recommendations for Testing, Managing, and Treating Hepatitis C Virus Infection. Hepatology. 2020; 71: $686-721$.

3. Kwo PY, Cohen SM, Lim JK. ACG Clinical Guideline: Evaluation of Abnormal Liver Chemistries. Am J Gastroenterol. 2017; 112: $18-35$.

4. Prati D, Colli A, Conte D, Colombo M. Spectrum of NAFLD and diagnostic implications of the proposed new normal range for serum ALT in obese women. Hepatology. 2005; 42: $1460-1$ author reply 1461 .

5. Donnan PT, McLernon D, Dillon JF, Ryder S, Roderick P, Sullivan F, et al. Development of a decision support tool for primary care management of patients with abnormal liver function tests without clinically apparent liver disease: A record-linkage population cohort study and decision analysis (ALFIE). Health Technol Assess. 2009; 13 (25): iii - iv, ix-xi.

6. Lilford RJ, Bentham L, Girling A, Litchfield I, Lancashire R, Armstrong D, et al. Birmingham and Lambeth Liver Evaluation Testing Strategies (BALLETS): a prospective cohort study. Health Technol Assess. 2013; 17 (28): i - xiv, 1 - 307.

7. Newsome PN, Cramb R, Davison SM, DIllon JF, Foulerton M, Godfrey EM, et al. Guidelines on the management of abnormal liver blood tests. Gut. 2018;67:6 -19.

8. Dufour DR, Lott JA, Nolte FS, Gretch DR, Koff RS, Seeff LB. Diagnosis and monitoring of hepatic injury. II. Recommendations for use of laboratory tests in screening, diagnosis, and monitoring. Clin Chem. 2000; 46: 2050 - 68.

9. Matsushita M, Komoda T. [Relationship between the effects of a high-fat meal and blood group in determination of alkaline phosphatase activity]. Rinsho Byori. 2011; 59: 923 - 9.

10. Castera L, Yuen Chan HL, Arrese M, Afdhal N, Bedossa P, Friedrich-Rust M, et al. EASL-ALEH Clinical Practice Guidelines: Non-invasive tests for evaluation of liver disease severity and prognosis. J Hepatol. 2015; 63: $237-64$.

11. McPherson S, Stewart SF, Henderson E, Burt AD, Day CP. Simple non-invasive fibrosis scoring systems can reliably exclude advanced fibrosis in patients with non-alcoholic fatty liver disease. Gut. 2010; 59:1265-9.

12. Angulo P, Hui JM, Marchesini G, Bugianesi E, George J, Farrell GC, et al. The NAFLD fibrosis score: A noninvasive system that identifies liver fibrosis in patients with NAFLD. Hepatology. 2007; 45: 846- 54

13. Boyum JH, Atwell TD, Schmit GD, Poterucha JJ, Schleck CD, Harmsen W cidence and Risk Factors for Adverse Events Related to Image-Guided Liver Biopsy. Mayo Clin Proc. 2016; 91:329 - 35.

14. Marchesini G, Day CP, Dufour JF, Canbay A, Nobili V, Ratziu V, et al. EASL-EASD-EASO Clinical Practice Guidelines for the management of non-alcoholic fatty liver disease. J Hepatol. 2016; 64: $1388-402$

15. Dasarathy S, Dasarathy J, Khiyami A, Joseph R, Lopez R, McCullough AJ. Validity of real time ultrasound in the diagnosis of hepatic steatosis: A prospective study. J Hepatol. 2009; 51:1061 - 7.

\section{PD Dr. med. Dr. phil. Christine Bernsmeier}

Gastroenterologie und Hepatologie

Clarunis - Universitäres Bauchzentrum Basel

Standort Universitätsspital Basel

Postfach

4002 Basel

christine.bernsmeier@clarunis.ch 